\title{
CHRONIC AUTOIMMUNE URTICARIA AND SJOGREN'S SYNDROME: CASE REPORT
}

Severino Correia do Prado Neto ${ }^{1,2,}$; Eduardo Martins Toniazzo ${ }^{1 ;}$; Fernanda Weber ${ }^{1 ;}$; Isabela Lisboa Rodrigues ${ }^{1}$; Jordanne Lopes Cordeiro²; Raiane Flávia Batista de Almeida1; Bruno Debona Souto²; Leana Ferreira Crispim ${ }^{1}$

1. Faculdade Morgana Potrich, Mineiros (GO), Brazil. 2. Centro Universitário de Mineiros, Mineiros (GO), Brazil.

*Corresponding author: severinopradoneto@gmail.com

\section{BACKGROUND}

Chronic urticaria (CU) is defined as the presence of urticaria with or without angioedema continuously or intermittently for at least 6 weeks. Approximately 12-21\% of patients with CU have some associated autoimmune disease (AID), and their clinical manifestations usually start 10 years after the diagnosis of $\mathrm{CU}$ and are more prevalent in women. Generally, cases of $\mathrm{CU}$ associated with AID have a higher remission rate of the cutaneous picture compared to $\mathrm{CU}$ alone. On the other hand, they negatively impact the patient's quality of life. Autoimmune thyroiditis is the main AID associated with CU. In addition to this, they highlight rheumatoid arthritis, lupus, type 1 diabetes mellitus, Sjögren's syndrome (RR15.17) and celiac disease. Sjögren's syndrome (SS) is an autoimmune and chronic rheumatic disease with glandular and extraglandular manifestations, more common in women between the fifth and sixth decade of life.

\section{CASE REPORT}

Female, 34 years old, diagnosed in May 2019 with CU without angioedema (Fig. 1) presenting high IgE, FAN NPFD 1/640 and FR $32(V R<8)$. She was using an antihistamine, with partial improvement. During rheumatological evaluation, she presented xerostomia, conjunctival hyperemia and bilateral tearing, xeroderma and symptoms of parotitis. Additional tests were requested: blood count, TGO, TGP, normal urea and creatinine; ESR $41 \mathrm{~mm}$; serum amylase $90 \mathrm{U} / \mathrm{L}$ (elevated); normal lipase; fasting blood glucose and normal Hb1AC; anti-Ro $240 \mathrm{U} / \mathrm{mL}$ (VR $<7 \mathrm{U} / \mathrm{mL}$ ); anti-CCP and anti-La NR; serologies for HIV, hepatitis B and C, VDRL and HTLV NR and serologies for toxoplasmosis, rubella and immune CMV. Salivary gland biopsy showing chronic nongranulomatous sialadenitis (Fig. 2). Ophthalmological evaluation proving dry eye. Hydroxychloroquine $400 \mathrm{mg} /$ day, prednisolone $10 \mathrm{mg} /$ day and lubricating eye drops were started. In January 2020, she had symptom improvement, in addition to normal ESR, weaning from corticosteroids and being maintained on antimalarial drugs. Report after the patient's consent.

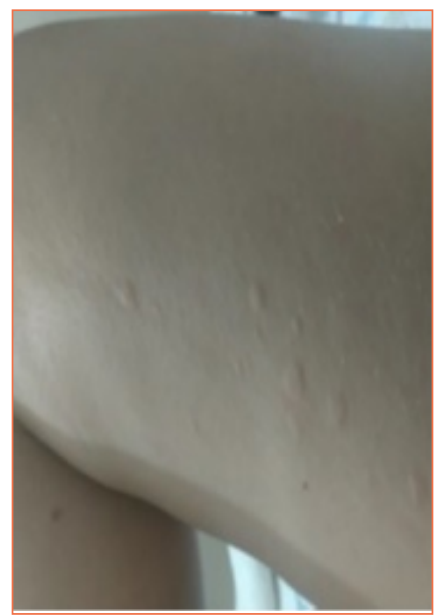

Figure 1. Urticarial lesions on the thigh. 


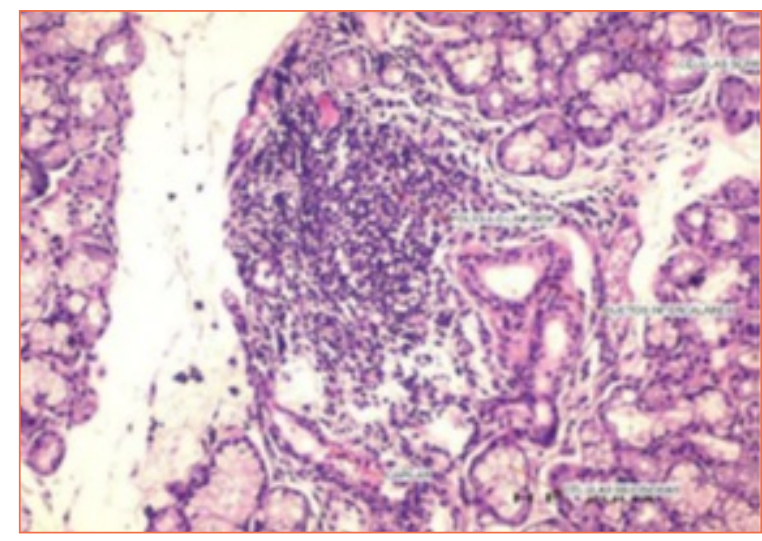

Figure 2. Salivary gland biopsy.

\section{CONCLUSION}

It is an atypical case because it is an SS in a young woman and the order of appearance of CU and SS, since the diagnoses were practically concomitant, differing from the literature. Patients with CU have an increased risk of SS, so this should be remembered in $\mathrm{CU}$ cases, especially in those without improvement with antihistamines, with dry symptoms and ANA present. 